\title{
Georg Forster oder die Reise um die Welt
}

Die Gründe, warum wir uns zumindest kurz mit Georg Forster beschäftigen sollten, sind sehr vielfältig. Zum einen wurde er über lange Zeit aus den deutschen Literaturgeschichten völlig verdrängt: sowohl als literarisch hochbegabter Reiseschriftsteller, trotz seines immensen Erfolges bei den Zeitgenossen, als auch vor allem als politischer Essayist und Denker. Als Jakobiner im Preußen und Deutschland der Restauration wurde er verabscheut und geächtet. Die von ihm verkörperte Traditionslinie wurde ganz bewusst aus den deutschen Literaturgeschichten ausgebürgert und sollte in Vergessenheit geraten, so als ob es sie nie gegeben hätte. Es bedurfte einiger Anstrengungen, dies zu verändern.

Georg Forster ist des Weiteren interessant, weil er in gewisser Weise jene Liste großer deutscher Reisender anführt, die nach ihm sicherlich Alexander von Humboldt als Schüler und Freund, aber auch Adalbert von Chamisso und so viele andere deutsche Reiseschriftsteller grundlegend beeinflusst haben. Denn Georg Forster vermochte es, mit Hilfe seiner stilistischen Mittel durch den Reisebericht eine ganz besondere Atmosphäre zu erzeugen, die das Lesepublikum auf eine wirkliche Entdeckungsreise mitnimmt. Denn sein Schreiben war kein Reise-Bericht als ein Notieren und Aufführen des Gesehenen und Untersuchten, sondern eine Reise-Literatur, welche die übergreifenden Zusammenhänge ebenso herausarbeitete wie sie die verschiedenen Beobachtungsweisen in einer ästhetischen Darstellungsweise zusammenlaufen ließ. Denn es ging ihm bei seinem ReiseSchreiben um ein Ganzes: um das Zusammenwirken möglichst vieler Faktoren und Aspekte in seiner Reise um die Welt. Dies macht ihn für uns Heutige so lebendig.

Schließlich gibt es kaum einen Reisebericht, der auch in seinen philosophischen Reflexionen in so ausgeprägtem Maße die Selbstreflexion auch über das eigene Tun sowie die Wahrnehmung des Anderen mit einschloss, also einen Grad an Selbstbesinnung und Selbstkritik einbaute, der zu seiner Zeit wahrlich außergewöhnlich war. Es geht mir also im Folgenden nicht darum, ein umfangreiches Bild Georg Forsters zu zeichnen, sondern anhand repräsentativer Beispiele herauszuarbeiten, inwieweit sein Schreiben für die weitere Entwicklung des Reiseberichts im 19. Jahrhundert von unschätzbarem Wert und als literarischer Orientierungspunkt von allergrößter Bedeutung ist.

Georg Forster lebte von 1754 bis 1794 und damit im Kernbereich der zweiten Phase beschleunigter Globalisierung. Dies hatte Folgen, denn er machte immer wieder mit Hinweisen und Bemerkungen auf all jene grundlegenden Veränderungen aufmerksam, die ihn zeitgenössisch umgaben. Dabei verfügte er über die Fähigkeit, sehr frühzeitig Entwicklungen zu bemerken, über welche die meisten seiner Zeitgenossen als unwichtig hinwegsahen. Denn man kann sehr 
gut inmitten des Wirbels einer Epoche leben, ohne jemals deren Charakteristika bemerkt zu haben. Doch bei Georg Forster war dies nicht der Fall.

Johann Georg Adam Forster wurde in Nasenhuben bei Danzig am 27. November 1754 als Ältestes von sieben Kindern geboren. Schon als Kind begleitete Georg Forster seinen Vater, den Pfarrer und späteren Naturforscher Johann Reinhold Forster (1729-1798) auf einer einjährigen Forschungsreise durch Russland. Diese Reise sollte den jungen Forster prägen, denn er erlebte die ungerechte Behandlung seines Vaters durch eine selbstgerechte, despotische Bürokratie hautnah mit.

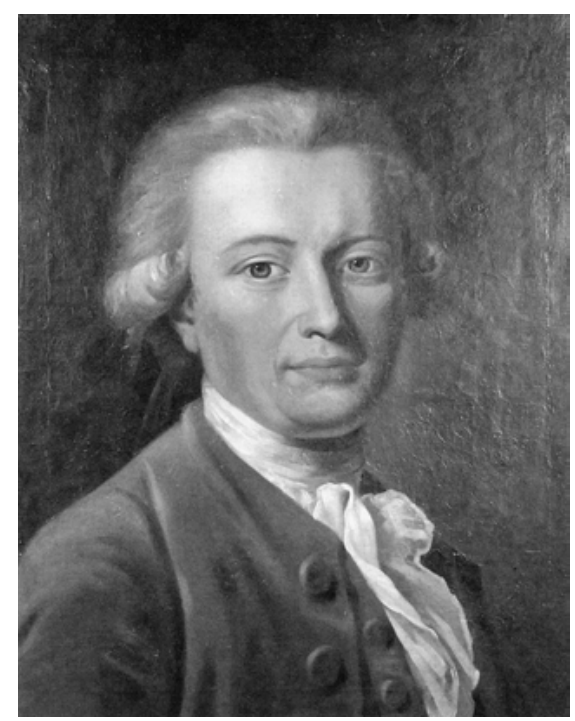

Abb. 68: Georg Forster (Nassenhuben, 1754 - Paris, 1794).

1766 wanderte seine Familie nach dem finanziellen Misserfolg dieser Expedition nach England aus, wo Forster als Übersetzer und Sprachlehrer die Familie miternähren musste. Rasch zeigte sich seine Sprachbegabung, schnell stellten sich erste Erfolge als Übersetzer unter anderem von Reiseberichten ein: Die Jahre in England prägten Forster außerordentlich stark. Von Beginn an erlebte er gleichsam die Welt aus der Perspektive der ökonomisch Abhängigen. 1772 wurde Forsters Vater für James Cooks zweite, dreijährige Weltreise als Naturforscher engagiert, wobei man ihm erlaubte, seinen Sohn als Gehilfen mitzunehmen. Dies war die Eintrittskarte in die Welt der Reiseliteratur für den noch jungen Georg Forster, der wie Kapitän Cook aus einfachen, ja ärmlichen Verhältnissen stammte. Forster war nach Beendigung der Weltumsegelung in der Lage, seine Erfahrungen während dieser Expedition binnen kurzer Zeit auf literarisch anspruchsvolle Weise niederzuschreiben, wobei er die Tagebücher 
seines Vaters mitauswertete. Diesem war jegliche Darstellung der Reise von James Cook vertraglich untersagt worden; dies aber galt für seinen Sohn nicht.

So konnte sein innerhalb von acht Monaten fertiggestelltes Werk, das im Wettlauf mit dem offiziellen Reisebericht von der Cook'schen Weltumsegelung entstand, bereits 1777 in englischer Sprache unter dem Titel A Voyage round the World erscheinen. Bereits kurze Zeit später - Georg Forster arbeitete wie besessen gegen die Zeit - lag sein Reisebericht in deutscher Sprache unter dem generischen Titel Reise um die Welt vor. Die Forster'sche Reisebeschreibung machte Furore.

Die brillante und elegante Reisebeschreibung machte den jungen Forster in ganz Europa schlagartig berühmt und in Deutschland zu einem der meistgelesenen Schriftsteller überhaupt. Auffällig war an Georg Forsters Stil, dass er in seinem durchaus der Aufklärung verpflichteten Werk genau das tat, was wir am literarischen Reisebericht auch in unseren theoretischen Auseinandersetzungen sehr früh in dieser Vorlesung bemerkt hatten: die Mischung zwischen verschiedenen Genera, die wir in der achten Dimension von Reiseliteratur gesondert untersucht hatten. So mischte Forster den eigentlichen Bericht mit der literarischen Erzählung, der wissenschaftlichen Abhandlung oder der philosophischen Reflexion, wobei sein eigentliches Hauptinteresse dem Menschen und seinen gesellschaftlichen Lebensformen galt. Von der dreijährigen Weltreise brachte Georg Forster eine ungeheure Fülle an Materialien und Dokumenten, aber auch eine Vielzahl von Zeichnungen nicht zuletzt auch unterschiedliche Vögel mit.

Fassen wir die weiteren Stationen in Georg Forsters kurzem Leben rasch zusammen. Von 1778 bis 1784 war Forster Professor für Naturgeschichte in Kassel, anschließend in Wilna in Polen. 1788 wurde er Bibliothekar in Mainz, das für ihn als linksrheinische Stadt sehr wichtig wurde. Er unternahm eine Reise mit dem noch sehr jungen Alexander von Humboldt und verfasste darüber die geographisch, wirtschaftlich-politisch und kulturell, vor allem aber ästhetisch-literarisch bedeutsamen Ansichten vom Niederrhein, von Brabant, Flandern, Holland, England und Frankreich, im April, Mai und Junius 1790, die reiseliterarische Maßstäbe setzten und ganz nebenbei den jungen Humboldt in seinem Schreiben sehr beeinflussten.

Georg Forster engagierte sich vehement für die Französische Revolution und für einen Anschluss der linksrheinischen deutschen Gebiete an die französische Republik. Seine Kontakte mit den Revolutionären in Paris vervielfachten sich. Als das 1793 von Frankreich besetzte Mainz von preußischen Truppen zurückerobert wurde, konnte Forster nicht mehr zurückkehren. Er starb 1794 völlig verarmt in Paris und wurde wegen seines Jakobinertums noch nach seinem Tode in Deutschland auf lange Jahrzehnte hin geächtet. Soweit und kurz gefasst seine Lebensgeschichte.

Lassen wir unseren kurzen Durchgang durch das Hauptwerk Georg Forsters, seine Reise um die Welt, mit einer Passage aus dem Vorwort beginnen, weil 


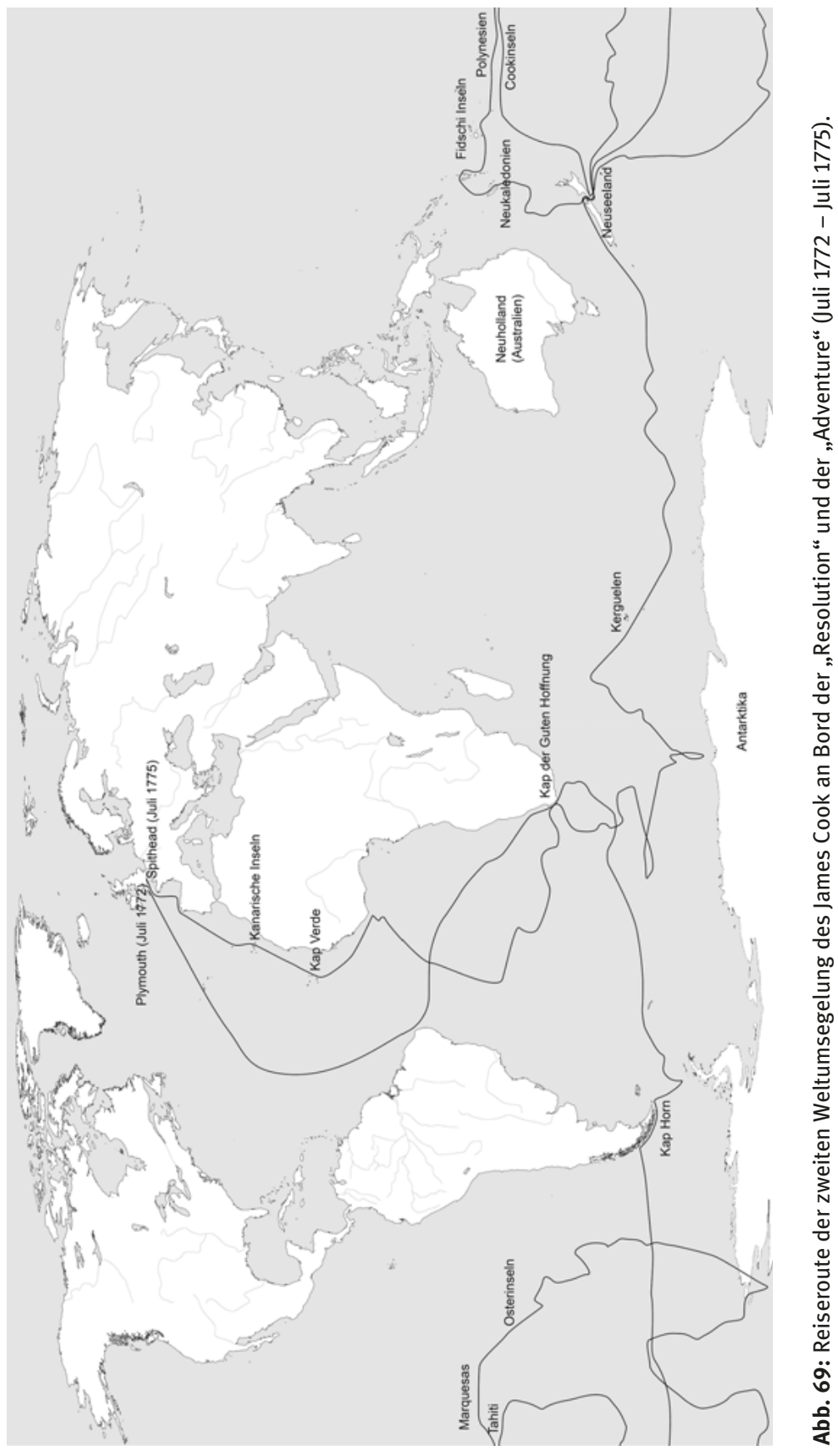


sie mir von großer Wichtigkeit für die Relevanz des Reiseberichtes überhaupt wie auch des Sammelns im Besonderen zu sein scheint. Im Vorwort positionierte sich der junge Mann, der freilich seinen Vater hinter sich wusste, recht selbstbewusst inmitten einer aufklärerischen Philosophie und Naturforschung, welche noch nach ihren Formen und Normen suchte. Deswegen sind die programmatischen Äußerungen, die sich hier finden lassen, von besonderer Bedeutung für unsere Frage nach der Epistemologie der von Forster praktizierten Wissenschaft und dem spezifischen Verhältnis zwischen Reisen und Schreiben.

Von Interesse ist dabei die Einschätzung der zeitgenössischen Entwicklungslinien, welche die Forschung im Zeitraum der europäischen Aufklärung prägten. Hören wir hierzu den Standpunkt des jungen Forster:

Die Philosophen dieses Jahrhunderts, denen die anscheinenden Widersprüche verschiedener Reisenden sehr misfielen, wählten sich gewisse Schriftsteller, welche sie den übrigen vorzogen, ihnen allen Glauben beymaßen, hingegen alle andre für fabelhaft ansahen. Ohne hinreichende Kenntniß warfen sie sich zu Richtern auf, nahmen gewisse Sätze für wahr an, (die sie noch dazu nach eigenem Gutdünken verstellten,) und bauten sich auf diese Art Systeme, die von fern ins Auge fallen, aber, bey näherer Untersuchung, uns wie ein Traum mit falschen Erscheinungen betrügen. Endlich wurden es die Gelehrten müde, durch Declamation und sophistische Gründe hingerissen zu werden, und verlangten überlaut, daß man doch nur Thatsachen sammeln sollte. Ihr Wunsch ward erfüllt; in allen Welttheilen trieb man Thatsachen auf, und bey dem Allem stand es um ihre Wissenschaft nichts besser. Sie bekamen einen vermischten Haufen loser einzelner Glieder, woraus sich durch keine Kunst ein Ganzes hervorbringen ließ; und indem sie bis zum Unsinn nach Factis jagten, verlohren sie jedes andre Augenmerk, und wurden unfähig, auch nur einen einzigen Satz zu bestimmen und zu abstrahiren; so wie jene Mikrologen, die ihr ganzes Leben auf die Anatomie einer Mücke verwenden, aus der sich doch für Menschen und Vieh nicht die geringste Folge ziehen läßt. ${ }^{1}$

In dieser durchaus polemisch eingefärbten Passage seiner 'Vorrede' griff Georg Forster mit der Gegenüberstellung von Philosophen und Sammlern in veränderter Form jenen Gegensatz von Reisenden und Daheimgebliebenen auf, der - wie wir bereits sahen - schon Jean-Jacques Rousseau und nach ihm Denis Diderot sowie Cornelius de Pauw, Antoine-Joseph Pernety oder GuillaumeThomas Raynal in eine weltweite Debatte verwickelt hatte. Georg Forster griff nicht direkt in diesen Disput ein - wozu er auch keinen Grund gehabt hätte -, zeigte sich aber auf der Höhe der Diskussionen seiner Zeit.

Seine Reflexion der epistemologischen Grundlagen von Erfahrung und Reisen stellte nicht nur die (denkarme) Empirie einem um Fakten wirklich nicht

1 Forster, Georg: Reise um die Welt. Herausgegeben und mit einem Nachwort von Gerhard Steiner. Frankfurt am Main: Insel Verlag 1983, S. 16 f. 
bekümmerten Systemdenken gegenüber, sondern zielte neben den Jägern und Sammlern auch auf jene, die sich in ihr Spezialistentum vergraben und 'das Ganze' aus den Augen zu verlieren drohen. Er selbst wollte sich durchaus als Reisenden verstehen, zugleich aber auch als einen Philosophen, dem es zwar nicht um ein Systemdenken, sehr wohl aber um die weltweiten Zusammenhänge ging. Man kann in dieser Passage durchaus das Bemühen erkennen, sich selbst als eine Art philosophe voyageur zu verstehen.

Das 1777 erstmals auf Englisch erschienene Werk, das zwischen 1778 und 1780 in deutscher Übersetzung und Bearbeitung erschien, ist in vielfacher Hinsicht die Schöpfung eines Multitalents, das nicht nur über erstaunlich breite wissenschaftliche, sondern auch literarische Kenntnisse und Fähigkeiten verfügte. Die schriftstellerische Kraft Forsters, aber auch seine Freude an intellektueller und emotionaler Neugierde und Inbesitznahme sind beeindruckend. Er war im Grunde eine Idealbesetzung, um die wissenschaftlichen Forschungsexpeditionen mit klar definierter Zielsetzung, wie sie James Cooks Reisen im britischen Kolonialinteresse darstellten, in eine literarische Form zu gießen. Georg Forster, der weitgehend Autodidakt war und sich viel von seinem Vater abgeschaut hatte, war dabei ein unruhiger Kopf, der auch die politischen Herausforderungen der überseeischen 'Begegnungen' mit anderen Menschen wie mit anderen Kulturen rasch begriff. Es ging ihm weder um ein abstraktes, von den Fakten losgelöstes Systemdenken nocch um ein Spezialistentum - in der Zeit von Mai 1968 hätte man von einem 'Fachidiotentum' gesprochen -, das in seinem eigenen beengten Wissenshorizont erstickt.

1766 war Louis-Antoine de Bougainville mit seiner Reise um die Welt in den Südpazifik aufgebrochen; doch mit James Cook riss England ohne jeden Zweifel die europäische Vorherrschaft an sich und profilierte sich als Seemacht auf allen Ozeanen unseres Planeten. Cook ergriff nach einem juristischen Schema und bestimmten europäischen Riten, die in nichts jenen des Columbus nachstanden, gleichzeitig von Neuseeland und von Australien Besitz. Die weißen Flecken auf dem Globus der europäischen Seemächte wurden geringer und verschwanden schließlich nahezu ganz: Selbst die entferntesten und verstecktesten Regionen der Erde begannen in einem unverändert nach Kolonien heißhungrigen Europa zunehmend bekannt zu werden. Die 1516 mit Thomas Morus entstandene Gattung der Utopie, welche die Projektion eines Landes in einen noch unbestimmten Raum vornimmt, wurde zunehmend durch die Uchronie ersetzt, in welcher sich ein Territorium nicht mehr in einen Raum, sondern in eine veränderte Zeit hinein projiziert.

Die Erde und ihr begrenzter Raum war den Europäern nun zunehmend bekannt; James Cook und mit ihm Georg Forster gehörten zu jenen, welche ihre Schrift in die zuvor den Europäern unbekannten Länder eintrugen. Wir nähern 
uns dem Höhepunkt der zweiten Phase beschleunigter Globalisierung, in denen England und Frankreich die Führungsmächte waren und in denen als Leitepidemie nicht mehr die Syphilis, sondern das Gelbfieber wütete. Die Entdecker und Eroberer aus Europa segelten nicht länger in den kleinen Nussschalen der Karavellen: Wesentlich zuverlässigere und schnellere Fregatten wurden zum globalisierenden Transportmittel Nummer 1 der europäischen Seemächte. Erneut begann sich die Welt zu wandeln. Und der europäische Reisebericht mit ihr.

Die grundlegende Erneuerung des Reiseberichts, die Vertiefung und intellektuelle Vergeistigung reiseliterarischen Schreibens hatte sich Georg Forster auf die Fahnen geschrieben - und er erreichte trotz widrigster Umstände sein Ziel auf beeindruckende Weise. Die zutiefst aufklärerische Grundhaltung orientierte den jungen Mann, der eigentlich nur der Gehilfe seines Vaters war, auf die Erfassung einer Gesamtheit, eines Ganzen hin, wie wir dies bereits im zurückliegenden Zitat sehr deutlich vor Augen geführt bekamen. Einzelne Fakten sollten nicht länger gleichsam in der Luft hängen, sondern auf organische und funktionale Weise miteinander verbunden sein. Es war nur mehr ein Schritt, bis man die Erde als zusammenhängendes System erfassen konnte.

Bei der Verwirklichung seiner Ziele kam ihm freilich zugute, dass er selbst keinerlei politische Rücksichten nehmen musste und nicht wie sein Vater unter der Zensur der britischen Admiralität stand. Er konnte seinen Reisebericht daher frei gestalten. Und Forster nutzte diese Chance. Im Mittelpunkt seines Schreibens, das wissenschaftliches und literarisches Lebenswissen miteinander verband, stand freilich immer wieder der Mensch selbst: der Mensch in seiner Vielfalt und Vielgestaltigkeit, der Mensch als Objekt und Subjekt zugleich der Reflexion und des eigenen Schreibens. In seinem Kosmos sah Alexander von Humboldt die Dinge ganz zutreffend: Mit Georg Forster hatte eine neue Ära wissenschaftlichen Reisens begonnen. Der Begleiter von James Cook pflegte die Anschaulichkeit einer lebendigen Darstellung, wie sie der Verfasser der Ansichten der Natur dann getreulich in den Fußstapfen des Autors der Ansichten vom Niederrhein praktizieren sollte.

Wir hatten im theoretischen Teil unserer Vorlesung die eindrucksvolle Passage aus Louis-Antoine de Bougainvilles Reise um die Welt näher analysiert, in welcher die Insel Tahiti aus der Zweidimensionalität der Meeresfläche aufzutauchen beginnt und zur Bühne einer sorgfältig konstruierten und komponierten Szenerie wird. Tahiti ist als locus amoenus seit Bougainvilles Beschreibung zweifellos stets mit dem Zusatz eroticus zu versehen. Denn eine solche Annäherung war fortan in den europäischen Literaturen eine wahre Reise auf die Insel der Liebenden, eine invitation au voyage für die Reise nach Kythera, der sagenumwobenen Insel der Verliebten, für die ein Watteau das wohl schönste Bildprogramm schrieb.

Tahiti stand fortan im Zeichen der Sinne, der Sinnlichkeit und der erotischen Reize. Die fêtes galantes, welche die Reise zur Liebesinsel verspricht, wurden auf 
diese Inselgruppe projiziert, die sich für die Zeitgenossen am Rande der bekannten Welt, weit weg im Südpazifik, befand. Der Mythos von der sagenumwobenen Insel der Liebenden und der freien Liebe hat seitdem zu Recht sehr gelitten. Der europäische Kolonialismus hat tiefe Spuren in den Gesellschaften des Archipels hinterlassen. Doch ihren verlockenden Reiz sollten diese Inselwelten noch zumindest bis in die Zeit eines Paul Gauguin behalten.

Ich kann der Versuchung nicht widerstehen, ihnen zumindest einen kleinen Einblick in Georg Forsters Reisebericht just am Ort von Bougainvilles großartiger Inszenierung zu geben. Unter der Feder des Deutschen, der sehr wohl von Bougainville wusste, beginnt dieser Aufenthalt auf Tahiti mit den folgenden denkwürdigen Worten: „Ein Morgen war's, schöner hat ihn schwerlich je ein Dichter beschrieben“2 - ein führwahr trefflicher Auftakt für eine Schilderung, die viel zur Südseetrunkenheit des europäischen Publikums beitragen sollte. Aber die Darstellung Georg Forsters war wie jene Bougainvilles alles andere als eindimensional.

Wählen wir dabei eine Passage, die sich im Übrigen im vielfachen Sinne einbauen lässt in die interkulturellen Diskurse, die im 18. Jahrhundert von Europa aus auf die Welt projiziert wurden. Sie werden dies gleich bemerken. Forster schildert eine Situation, in der gerade James Cook und seine Offiziere das Schiff verlassen haben und so die Matrosen sozusagen alleine 'zuhause' an Bord sind. Was in solchen Fällen einer sturmfreien Bude auf Tahiti passieren kann, wird im folgenden Ausschnitt aus der Reise um die Welt, die vom 13. Juli 1772 bis zum 30. Juli 1775 stattfand, gezeigt:

Während dieser Zeit war das Schiff mit einer Menge von Canots umringt, die außer allerhand Kräuterwerk, auch große Quantitäten einländischen Zeugs verhandelten. So gar auf den Verdecken wimmelte es von Indianern, und unter selbigen gab es verschiedene Frauenspersonen, die sich ohne Schwierigkeiten den Wünschen unsrer Matrosen überließen. Einige von denen, die dieses Gewerbe trieben, mochten kaum neun oder zehn Jahr alt seyn und hatten noch nicht das geringste Zeichen der Mannbarkeit an sich. So frühzeitige Ausschweifungen scheinen einen sehr hohen Grad von Wollust anzudeuten und müssen im Ganzen allerdings Einfluß auf die Nation haben. Die natürlichste Folge davon, die mir auch sogleich in die Augen fiel, bestand darinn, daß das gemeine Volk, zu welchem alle diese liederlichen Weibsbilder gehören, durchgehends von kleiner Statur war. Nur wenige einzelne Leute aus demselben, waren von mehr als mittlerer Größe; die übrigen waren alle darunter - ein Beweis, daß die Meynung des Grafen Büffon, über die frühzeitige Vermischung beyder Geschlechter (S. dessen Hist. naturelle) sehr gegründet ist. Sie hatten unregelmäßige, gemeine Gesichtszüge, aber schöne, große Augen, die durchgehends sehr lebhaft waren; nächst diesen ersetzte auch ein ungezwungenes Lächeln

2 Forster, Georg: Reise um die Welt, S. 241. 


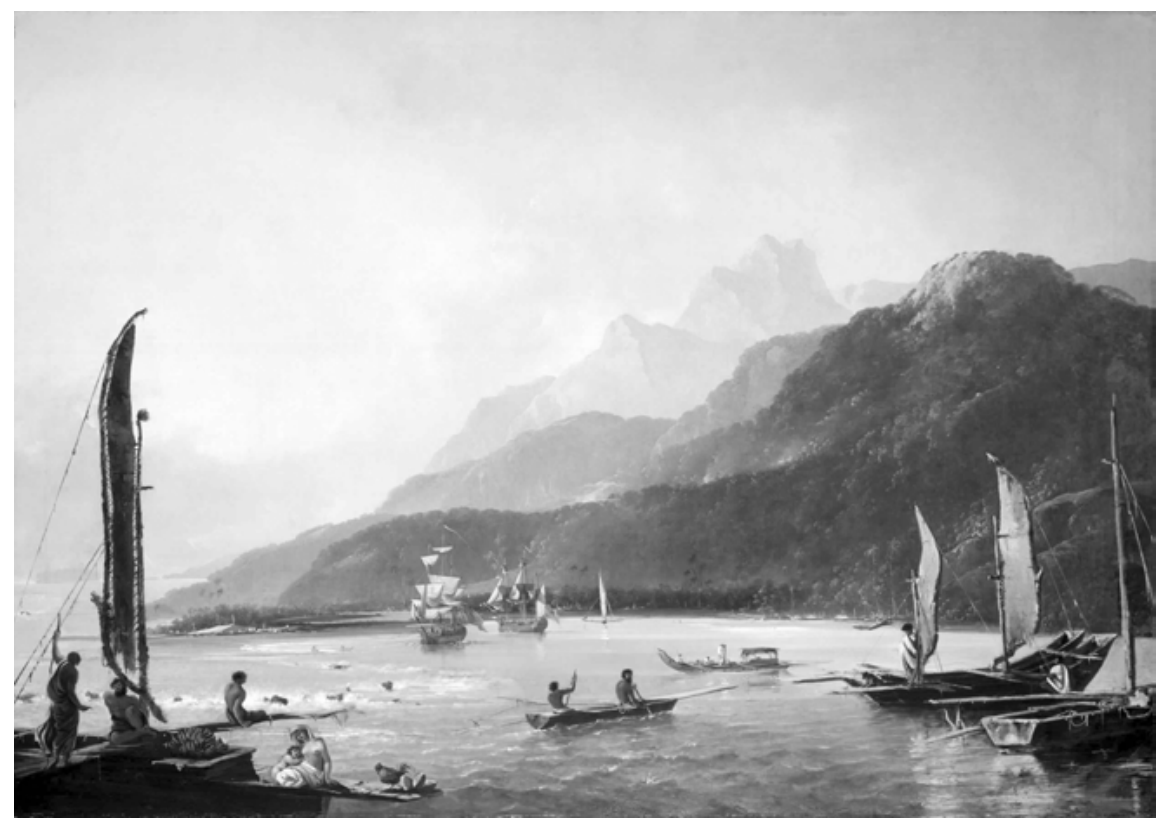

Abb. 70: „[A] view of Maitavie Bay, [in the island of] Otaheite [Tahiti]“, Landschaftsmalerei von William Hodges, 1776.

und ein beständiges Bemühen zu gefallen, den Mangel der Schönheit so vollkommen, daß unsre Matrosen ganz von ihnen bezaubert waren und auf die leichtsinnigste Weise von der Welt, Hemder und Kleider weggaben, um sich diesen neuen Mätressen gefällig zu bezeigen. Die ungekünstelte Einfalt der Landes-Tracht, die den wohlgebildeten Busen und schöne Arme und Hände unbedeckt ließ, mochte freylich das ihrige beytragen, unsre Leute in Flammen zu setzen; und der Anblick verschiedner solcher Nymphen, davon die eine in dieser, jene in einer andern verführerischen Positur behend um das Schiff herschwammen, so nackt als die Natur sie gebildet hatte, war allerdings mehr denn hinreichend, das bisschen Vernunft ganz zu blenden, das ein Matrose zu Beherrschung der Leidenschaften etwa noch übrig haben mag. ${ }^{3}$

Zunächst einmal ist es aufschlussreich, daß Georg Forster auf Tahiti von 'Indianern' spricht. Diese Tatsache zeigt nicht zuletzt, wie sehr sich die Eindrücke des Umgangs mit außereuropäischen Völkern vermischen können und wie stark vor allem die Präsenz all jener Diskurse ist, die sich seit Ausgang des 15. Jahrhunderts in Europa über die außereuropäische Welt herausgebildet

3 Forster, Georg: Reise um die Welt, S. 250 f. 
haben. Die Sichtweise des Columbus zeitigte Folgen, die bis ins 18. Jahrhundert, aber auch bis in unsere Gegenwart andauern.

Deutlich wird in dieser Passage von Forsters Reise um die Welt nicht nur, dass die sozialen Unterschiede und das Standesbewusstsein des angehenden Reiseschriftstellers, der nicht als einfacher Matrose an Bord war, sondern als ein Naturforscher, eine klare Abgrenzung und Ausgrenzung der Matrosen vornimmt, welche gleichsam aus dem Reich der Vernunft ausgebürgert und von der intellektuellen Elite des Schiffes klar geschieden werden. Anders als das gemeine Schiffsvolk vermag der Reiseschriftsteller selbst seine Sinne so sehr zusammenzunehmen, dass er diese Szenerie nicht nur von einer gleichsam höheren Warte aus distanziert schildern kann und ganz nebenbei den Reizen der tahitianischen Schönen nicht unterliegt, sondern vielmehr eine Vielzahl kritischer Beobachtungen und Bemerkungen niederschreibt oder Vergleiche mit der Antike anstellt, welche die tahitianische Gegenwart mit einem Hauch von antiker Sinnenfreude umgeben. Auch dies gehört zur Selbstinszenierung eines reisenden Ich, das von einem gereisten Ich fast nebenbei mit Attributen ausgestattet wird, welche seine Kompetenz unterstreichen. Das reisende Ich behält folglich einen kühlen Kopf und schenkt weder Hemd noch Hose her.

Die jungen tahitianischen Frauen erscheinen in einer sehr ambivalenten Weise als Nymphen und zugleich als Prostituierte, als Nymphomaninnen und zugleich als unschuldige Naturmenschen, als Hässliche von kleiner Statur und als Schöne mit wohlgestalteten Proportionen, so dass der gesamten Passage ein hohes Maß an Ambivalenz und Oszillation zukommt. Das erzählende Ich und das erzählte Ich scheinen sich nicht auf einen Standpunkt einigen zu können. Sind die femmes naturelles von Tahiti nun bewunderns- oder verachtenswert?

Zweifellos ist auch bei Georg Forster Tahiti der Ort der Lüste, ja der Ort der Wollüste, die hier zugleich in einen medizinisch-biopolitischen Diskurs eingebaut werden, der in Buffons Histoire naturelle seinen wissenschaftlichen Bezugspunkt besitzt. Europäische Sexualvorstellungen werden in einen wissenschaftlich-kritischen Diskurs überführt. Minderjährige tahitianische Mädchen werden dabei im europäischen Diskurs von der Geschlechtlichkeit gleichsam ausgeschlossen; und zugleich werden sie von denselben Europäern für deren Sexualpraktiken sehr wohl benutzt. Man kann die ganze Wucht und Gewalt erkennen, mit welcher ein Gesellschafts- und Moralsystem auf ein anderes herniederfährt. Und in der Tat sollten von der tahitianischen Gesellschaftsordnung nur noch Scherben übrig bleiben.

Auch in dieser Passage Georg Forsters finden wir das Motiv der schönen unbekannten Frau wieder, das die Europäer auf deren eigenem Schiff heimsucht und obsessiv so lange verfolgt, bis sie - wohlgemerkt aus der übergeordneten europäischen Perspektive - alle Vernunft fahren lassen und zu Gefangenen ihrer 
eigenen Natur werden. Wie hätte man den sexuell ausgehungerten Männern an Bord, aus gesellschaftlich niederen Schichten stammend, Einhalt gebieten können? Es wird nicht einmal der Versuch hierzu unternommen.

So darf denn auch bei diesen Überlegungen der Verweis auf die Kraft der Natur im Gegensatz zur Vernunft nicht fehlen, jenen grundlegenden Polen, an denen sich die europäische Aufklärung abarbeitete. Vernunft ist schließlich gesellschaftlich geprägt und determiniert, so will es scheinen. Dass Georg Forster, selbst noch reichlich jung an Jahren und an Bord, nicht umhin konnte, bei diesen Mädchen und jungen Frauen widerstrebend eine ausgeprägte, von den Augen ausgehende Schönheit zu konstatieren, macht uns zum einen darauf aufmerksam, dass hier erneut - wie schon in seiner Schilderung der Menschen auf den Kapverdischen Inseln - europäische Schönheitsbegriffe ganz selbstverständlich Verwendung finden. Und dass zum anderen aber auch die Möglichkeit und Fähigkeit besteht, die Schönheit eines Körpers ausgehend von bestimmten Punkten - hier insbesondere auch den Augen - zu rekonstruieren, also auch hässliche Attribute gleichsam überspielen oder überstrahlen zu lassen. Dabei ist es spannend zu beobachten, dass das reisende Ich die Schönheit gerade anhand der Augen 'verleiht', welche einerseits den Geschlechtsmerkmalen fern und andererseits den Sinn assoziieren, durch welchen all diese Eindrücke auf den jungen Mann einprasseln. Gleichwohl wird er seine distanzierte Beobachterposition nicht verlassen.

Nun gut: Nicht nur die Matrosen an Bord der Schiffe James Cooks, sondern wohl auch andere Teile der Besatzung werden die Kautelen unseres Beobachters wenig gekümmert haben, konnten sie doch hier Geschlechterbeziehungen ausleben, die in Europa selbst in ihren Sphären in den Häfen der Welt wesentlich stärker normiert und tabuisiert waren. Hätte es sich um reine Prostitution gehandelt, so hätten die europäischen Männer nicht so rasch den Verstand verloren und wären nicht so in Flammen aufgegangen, wie dies der Reisebericht andeutet. Zumindest darf man diese Vermutung an dieser Stelle aussprechen, auch wenn sich hier die Geschlechterordnung und die koloniale (Unter-)Ordnung grundlegend überkreuzen und wechselseitig verstärken. Die Ambivalenz von wissenschaftlichem Diskurs und tatsächlicher kolonialer Praxis bei den Europäern ist freilich evident.

Doch kehren wir zu den allgemeinen wissenschaftlichen Fragestellungen zurück, die Georg Forsters Reise um die Welt aufwerfen. Für Forster wird Wissenschaft letztlich nicht um ihrer selbst willen betrieben, sondern muss einem übergeordneten Ziele dienen: sei es dem Wohle der Menschheit insgesamt, sei es ihrer Erforschung und geschichtlichen Entwicklung in einem historiographischanthropologischen oder geographischen Sinne. An derlei Zielen orientiert sich auch Georg Forsters ausgeprägte Methode des Vergleichs, die von grundlegender Bedeutung ebenso für sein eigenes Werk wie später für jenes eines Alexander von Humboldt ist. Cornelius de Pauws Warnung, dass in den europäischen 
Wissenschaften immer auch der Keim der Zerstörung ihrer jeweiligen Objekte ruhe, sei an dieser Stelle freilich noch einmal erwähnt.

Doch in der Ethik beider europäischer Wissenschaftler war die Verpflichtung auf das große Ganze und dessen Rechtfertigung mit dem Verweis auf eine allgemeine Geschichte der Gattung Mensch letztlich unantastbar. Ihre Intention war es zu keinem Zeitpunkt, den untersuchten Kulturen Schaden zuzufügen oder etwa zur kolonialen Ausplünderung durch die europäischen Mächte beizutragen. Es verwundert daher nicht, dass Georg Forster in seiner Reise um die Welt stets versuchte, die von ihm untersuchten Phänomene in eine Geschichte globalen Zuschnitts zu integrieren. In diesen Versuchen der Entwicklung einer generellen Sichtweise kam er zu wahrlich bemerkenswerten Einschätzungen, mit denen wir uns abschließend noch beschäftigen wollen.

Verlassen wir dazu sein Opus Magnum, das ihn berühmt machte, und behandeln wir kurz seine kleineren Schriften. In seiner 1791 verfassten Abhandlung Die Nordwestküste von Amerika, und der dortige Pelzhandel hat Georg Forster, der rasch beim deutschsprachigen Lesepublikum zu einem weithin bekannten und bewunderten Spezialisten für die Geschichte der europäischen Expansion im 18. Jahrhundert geworden war, gleich zu Beginn eine folgenreiche Einschätzung des damaligen europäischen Kenntnisstandes gegeben:

Der Zeitpunkt nähert sich mit schnellen Schritten, wo der ganze Erdboden dem Europäischen Forschungsgeiste offenbar werden und jede Lücke in unseren Erfahrungswissenschaften sich, wo nicht ganz ausfüllen, doch in so weit ergänzen muß, daß wir den Zusammenhang der Dinge, wenigstens auf dem Punkt im Äther den wir bewohnen, vollständiger übersehen können. Bald ist es Nationaleitelkeit, bald politisches Interesse, Spekulation des Kaufmanns oder Enthusiasmus für Wahrheit, was auf jenes Ziel hinarbeitet und dem wichtigen Endzwecke mit oder ohne Bewußtseyn dienen muß. Wie greifen alsdann die Räder des großen kosmischen Mechanismus so wunderbar in einander! [...]

Hier beginnt eine neue Epoche in der so merkwürdigen Geschichte des Europäischen Handels, dieses Handels, in welchen sich allmählig die ganze Weltgeschichte aufzulösen scheint. Hier drängen sich dem Forscher so viele Ideen und Thatsachen auf, daß es die Pflicht des Herausgebers der neuen Schifffahrten und Landreisen in jener Gegend mit sich zu bringen scheint, alles, was auf die Kenntniß derselben Beziehung hat, in einen Brennpunkt zu sammeln und zumal einem Publikum, wie das unsrige, welches nur einen litterarischen, mittelbaren Antheil an den Entdeckungen der Seemächte nehmen kann, die Übersicht dessen, was bisher unternommen worden ist, und das Urtheil über die Wichtigkeit dieser ganzen Sache zu erleichtern. ${ }^{4}$

4 Forster, Georg: Die Nordwestküste von Amerika, und der dortige Pelzhandel, in Ders.: Werke. Sämtliche Schriften, Tagebücher, Briefe. Bd. 5: Kleine Schriften zur Völker- und Länderkunde. Herausgegeben von Horst Fiedler, Klaus Georg Popp, Annerose Schneider und Christian Suckow. Berlin: Akademie-Verlag, 1985,S. 390-395. 
Am Ineinandergreifen der „Räder des großen kosmischen Mechanismus“ hatte Georg Forster durch seine Teilnahme an der zweiten Weltumsegelung von James Cook zweifellos seinen Anteil. Die französischen und britischen Weltumsegelungen setzten den Maßstab jener Erforschung und Erkundung, aber auch Vermessung und Verteilung der Welt, an denen das deutschsprachige Publikum - wie Forster sehr bewusst betont - keinen direkten Anteil hat. Noch war die Kolonialpolitik in den deutschen Landen nur rudimentär und bruchstückhaft, so dass das deutsche Publikum vor allem einen intellektuellen Anteil am Ausgreifen wie am Ausgriff Europas in dieser Phase der Weltgeschichte hatte. Aber auch dieser Anteil war, zusätzlich zu jenem deutscher Kaufleute und Händler im großen kosmischen Mechanismus der Globalisierung, keineswegs gering. Und Georg Forster wusste dies sehr genau.

Sein zeitgenössischer Kommentar zur europäischen Expansion der zweiten Hälfte des 18. Jahrhunderts, die wir im Kontext unserer Vorlesung wie auch der transarealen Studien insgesamt auch als zweite Phase beschleunigter Globalisierung bezeichnet haben, macht deutlich, wie endlich der planetarische Raum und wie absehbar seine detaillierte Erkundung geworden zu sein schienen. Bald also wurden die weißen Flecken auf den Landkarten und Globen der europäischen Herrscher, der wissenschaftlichen Gesellschaften und der großen Handelshäuser und Kaufleute kleiner und kleiner. Die Metaphorik der schnellen Schritte, mehr aber noch jener „Räder des großen kosmischen Mechanismus“, die sich immer schneller drehten und für den Menschen immer erkennbarer ineinandergriffen, verdeutlicht den Glauben an eine auf Fortschritt, Beherrschbarkeit und (Erfahrungs-) Wissenschaften setzende Vernunft, die jene des „Europäischen Forschungsgeiste[s]“ ist. Georg Forster stand mit beiden Beinen fest auf dem Fortschrittsglauben der europäischen Aufklärung.

Der berühmte Verfasser der Reise um die Welt, mit dem Alexander von Humboldt 1790 eine mehrmonatige Reise unternahm, welche die beiden unter anderem nach London und auf dem Rückweg ins revolutionäre Paris führte, gehört in Deutschland zweifellos zu jenen Autoren, die am meisten für die Entwicklung dieser im wahrsten Sinne des Wortes auf Erfahrungswissen beruhenden Kenntnisse einer sich verändernden und an Bedeutung noch zunehmenden Reiseliteratur am Ausgang des 18. Jahrhunderts beitrugen. ${ }^{5}$ Er war zweifellos einer der Protagonisten und wichtigsten Verkünder der europäischen Expansion.

5 Vgl. hierzu Wuthenow, Ralph-Rainer: Die erfahrene Welt. Europäische Reiseliteratur. im Zeitalter der Aufklärung. Mit zeitgenössischen Illustrationen. Frankfurt am Main: Insel Verlag 1980; sowie Wolfzettel, Friedrich: 'Ce désir de vagabondage cosmopolite.' Wege und Entwicklung des französischen Reiseberichts im 19. Jahrhundert. Tübingen: Max Niemeyer Verlag 1986. 
Doch die Räder dieser kosmischen Maschinerie drehten sich derart schnell, dass sie - wie wir in kinematographischer Metaphorik hinzufügen könnten - bisweilen 'stillzustehen' schienen. Die letzten verbliebenen Lücken des Erdraumes ${ }^{6}$ waren bald schon ausgefüllt: War es dann nicht bald auch die Zeit als Zeit-Raum wie als Raum-Zeit? Georg Forster, den Alexander von Humboldt mehrfach zu Recht nicht nur als seinen Freund, sondern als seinen Lehrmeister bezeichnete, hielt mit Blick auf die Erforschung der Nordwestküste des amerikanischen Doppelkontinents fest, wie sehr sich all dies in eine allgemeine Geschichte der Menschheit einfügte. Diese Menschheitsgeschichte aber begann sich in den Augen Forsters grundlegend zu verändern.

Die zentrale Beobachtung Georg Forsters war erhellend und provokativ zugleich: Löste sich also die gesamte Weltgeschichte in den Welthandel auf? Kam die allgemeine Geschichte der Menschheit vielleicht dadurch zu einem Stillstand, dass sie durch Vervielfältigung von Kommunikation ersetzt wurde? Diese Darstellung einer Epoche der Nachzeitlichkeit will uns aus heutiger Sicht als geradezu nach-modern erscheinen. Konstatierte Forster in diesen Zeilen ein Ende der Geschichte und den Beginn einer Post-Temporalität?

Diese Passage bringt aber nur ein verändertes, beschleunigtes Zeitempfinden zum Ausdruck, das nicht bloß den Verfasser der Reise um die Welt am Ausgang des 18. Jahrhunderts - und keineswegs allein auf Grund der Erfahrung der Französischen Revolution - ergriffen hatte. Georg Forster war nicht Jules Michelet: Der französische Historiker hatte in seiner Sichtweise der Geschichte die Epochenerfahrung der Französischen Revolution als Orientierungspunkt gesetzt. Für ihn war alles, was danach kommen sollte, nur eine Zeit nach dem Ende der Geschichte - schaler Abklatsch eines Ereignisses von menschheitsgeschichtlichem Rang.

Nicht so Forster, obwohl auch für ihn die Französische Revolution ein einschneidendes Epochenereignis und Epochenerlebnis war. Die prägnante Charakterisierung dieser weltgeschichtlichen Epoche der europäischen Expansion, ihrer weltweiten Handelsverflechtungen und ihrer wissenschaftlichen wie 'literarischen' Aufarbeitung ließ keinen Zweifel daran aufkommen, dass es sich im Sinne Georg Forsters um einen unaufhaltsamen, unabänderlichen Prozess

6 Forster, Georg: Die Nordwestküste von Amerika, und der dortige Pelzhandel, S. 393: „So ist nicht nur unsere jetzige physische und statistische Kenntniß von Europa zur Vollkommenheit gediehen, sondern auch die entferntesten Welttheile gehen allmählig aus dem Schatten hervor, in welchem sie noch vor kurzem begraben lagen.“ Auch hier kommt das Licht aus Europa, die Kenntnisse und Selbstkenntnisse außereuropäischer Völker liegen noch im Schatten europäischer Interessen. Forster war zweifellos ein privilegierter Beobachter dieser gesamten Entwicklung. 
handelte, der von den unterschiedlichsten Interessen und Spekulationen „mit oder ohne Bewußtseyn“ dieser Entwicklungen vorangetrieben werde. Georg Forster besaß folglich ein klares Epochenbewusstsein seiner Zeit, zweifellos eine Frucht seiner Reisen, die ihm immer wieder andere Perspektiven auf seine Zeitepoche ermöglichten. Es waren diese Bewegungen und ihre ständigen Reflexionen, die ihn von seinen Zeitgenossen nicht nur in Deutschland unterschieden.

Dabei war eine intentionale Lenkung dieses Prozesses nicht vonnöten: Alles lief in der Logik der „kosmischen Maschinerie“ seltsam verselbständigt bis zum Erreichen seines 'Endzwecks' ab. So finden wir bei Georg Forster ein sehr geschärftes Bewusstsein für jene Prozesse, die wir als Phänomene einer zweiten Phase beschleunigter Globalisierung bezeichnen können. Er erkannte, dass die Weltumsegelungen der Bougainville, Cook oder Lapérouse weitaus mehr waren als Reisen zur Erforschung und Vermessung unseres Planeten: Sie waren vielmehr das Zeichen einer epochalen Veränderung. Die Zeit war nicht mehr zurückzudrehen: Sie verlief in eine nunmehr offene Zukunft.

In Georg Forsters Reise um die Welt wird die Untersuchung des Menschen als integraler Bestandteil der Erforschung der Natur betrachtet, wie schon Peter Schmitter ${ }^{7}$ sehr zutreffend bemerkte. Eine naturkundliche Forschung scheint für Forster letztlich nur mit Blick auf den Menschen interessant und aufschlussreich zu sein: Wichtig ist ihm vor allem, einen Beitrag zur Kenntnis des Menschengeschlechts und dessen Geschichte zu leisten. Letztlich, so Schmitter, sei es Forster darum gegangen, durch vollkommenere Kenntnisse zur Vervollkommnung des Menschen selber beizutragen.

Diesem Ziel dienen auch seine wiederholten Reisen, in denen immer wieder die Idee menschlicher Perfektibilität aufscheint. Diese Perfektibilität - ein Neologismus des 18. Jahrhunderts, der auf Jean-Jacques Rousseau zurückgeht - ist dabei für Forster stets ein Prinzip, das der Natur selbst schon inhärent ist. Wir stoßen hier nicht zuletzt auf den Evolutionsgedanken, der auch das Werk Alexander von Humboldts durchziehen wird, letztlich aber erst in seiner radikalisierten Form bei Charles Darwin 1859 in seinem berühmten On the Origins of Species by Means of Natural Selection ausgefaltet und wissenschaftlich durchbuchstabiert werden wird.

All dies hat in ganz wesentlicher Weise mit jener kosmopolitischen Tradition $\mathrm{zu}$ tun, innerhalb derer sich Georg Forsters eigenes Tun und Denken

7 Vgl. Schmitter, Pegter: Zur Wissenschaftskonzeption Georg Forsters und dessen biographischen Bezügen zu den Brüderen Humboldt. Eine Vorstudie zum Verhältnis von allgemeiner Naturgeschichte, physischer Weltbeschreibung und allgemeiner Sprachkunde. In: Naumann, Bernd / Plank, Frans / Hofbauer, Gottfried / Hooykaas, Reiner (Hg.): Linguistics of Geology. Amsterdam: benjamins 1992, S. 91-124. 
plazierte, eine Tradition, die sich im Spielraum des Weltbürgertums des 18. Jahrhunderts bewegte. Dabei gibt es eine Grundströmung, welche sicherlich für Georg Forster gilt, nämlich Kosmopolitismus als Form des Universalismus: beruhend auf der grundlegenden Überzeugung, dass alle Menschen bestimmter Dinge teilhaftig sind und diese jenseits des Nationalen einer globalen Ordnung zuweisen. Sehen wir von Alexander von Humboldt einmal ab, der den Kosmopolitismus entschlossen tief ins 19. Jahrhundert projizierte, so gehört Georg Forster zweifellos in jene Epoche, die den Höhepunkt eines Kosmopolitismus signalisierte, welcher dann um 1800 sehr rasch in einer Art Gegenpendelbewegung verfallen und zerfallen sollte. Forster ist auch in dieser Hinsicht im umfänglichen Sinne noch ein Mann des 18. Jahrhunderts.

Dabei gab es in Deutschland am Ausgang des Jahrhunderts der Aufklärung laut Pauline Kleingeld ${ }^{8}$ sechs verschiedene Varietäten von Kosmopolitismus, die ich hier nur kurz aufzählen kann, die aber den Horizont der Möglichkeiten abstecken. Man unterscheidet erstens den moralischen Kosmopolitismus, zweitens die Vorschläge zur Veränderung der internationalen politischen (und rechtlichen) Ordnung, drittens den kulturellen Kosmopolitismus, der die Rolle eines globalen kulturellen Globalismus hervorhob, viertens einen ökonomischen Kosmopolitismus, der auf die Entwicklung eines globalen freien Marktes abzielte, auf dem alle Menschen potentiell freie und gleiche Handelspartner sein könnten, fünftens einen juristischen Kosmopolitismus sowie schließlich sechstens das romantische komsopolitische Ideal einer Menschheit, die durch Glaube und Liebe miteinander verbunden ist und eine Einheit bildet. In diesen verschiedenen Spielarten von Kosmopolitismus sehen wir leicht die Vorläufer aktueller Debatten und Beziehungen, die auch noch unsere globalisierte Welt insbesondere von Kultur und Wirtschaft bestimmen. Doch nicht alle Blütenträume des Kosmopolitismus reiften - vor allem gab es im 19. Jahrhundert einen politischen Gegenschlag, der mit dem Aufstieg des Nationalismus rasch dem aufkommenden Kosmopolitismus im politischen Bereich den Garaus machte. 'Kosmopolit' verkam rasch auch zu einem Schimpfwort, das nicht zuletzt auch im sozialistischen Lager fortan sehr wirkkräftig war.

Doch ich möchte an dieser Stelle die ausführlich von Pauline Kleingeld diskutierten Varietäten des Kosmopolitismus nicht mit Ihnen durchdeklinieren. Denn unser Ziel ist es nicht, wichtige Aspekte des Epochenwandels um $1800 \mathrm{zu}$ beschreiben, sondern zu verstehen, inwiefern sich die historischen und kulturellen Kontexte an der Wende zum 19. Jahrhundert veränderten. Entscheidend für uns

8 Vgl. Kleingeld, Pauline: Six Varieties of Cosmopolitanism in Late Eigteenth-Centruy Germany. In: Journal of the History of Ideas (Baltimore) LX, 3 (July 1999), S. 505-524. 
scheint mir abschließend, dass viele dieser Aspekte in der Tat im Werk Georg Forsters zum Ausdruck kommen und in seinen Schriften transportiert werden.

Am Ende unserer (ich hoffe doch) repräsentativen Beschäftigung mit dem großen deutschen Reisenden des 18. Jahrhunderts möchte ich Ihnen nicht die sich unmittelbar an die Rückkunft in England anschließende Schlusspassage seiner Reise um die Welt vorenthalten. Sie versucht, bevor sie in ein PetrarcaZitat ausmündet, eine Art panoramatischen Überblick zu bieten, wobei es hier erneut und offenkundig die Menschen und ihre Varietäten sind, die den Reisenden besonders beeindruckt haben und beschäftigen:

So vollendeten wir, nachdem wir unzählige Gefahren und Mühseligkeiten überstanden, eine Reise, die drey Jahre und achtzehn Tage gedauert hatte. [...] In andern Jahreszeiten hatten wir das Stille-Meer innerhalb der Wende-Zirkel befahren; und daselbst den Erdbeschreibern neue Inseln, den Naturkundigern neue Pflanzen und Vögel, und den Menschenfreunden insbesondere, verschiedene noch unbekannte Abänderungen der menschlichen Natur aufgesucht. In einem Winkel der Erde hatten wir, nicht ohne Mitleid, die armseligen Wilden von Tierra del Fuego gesehn; halb verhungert, betäubt und gedankenlos, unfähig sich gegen die Rauhigkeit der Witterung zu schützen, und zur niedrigsten Stufe der menschlichen Natur bis an die Gränzen der unvernünftigen Thiere herabgewürdigt. In einer andern Gegend hatten wir die glücklichern Völkerschaften der Societäts-Inseln bemerkt; schön von Gestalt und in einem vortreflichen Clima lebend, welches alle ihre Wünsche und Bedürfnisse befriedigt. Ihnen waren schon die Vortheile des geselligen Lebens bekannt; bey ihnen fanden wir Menschenliebe und Freundschaft; ihnen war es aber auch zur Gewohnheit geworden, der Sinnlichkeit bis zur Ausschweifung Raum zu geben. Durch die Betrachtung dieser verschiedenen Völker, müssen jedem Unpartheyischen die Vortheile und Wohlthaten, welche Sittlichkeit und Religion über unsern Welttheil verbreitet haben, immer deutlicher und einleuchtender werden. Mit dankbarem Herzen wird er jene unbegreifliche Güte erkennen, welche ihm ohne sein Verdienst einen wesentlichen Vorzug über so viele andre Menschen gegeben, die ihren Trieben und Sinnen blindlings folgen, denen die Tugend nicht einmal dem Namen nach bekannt, und für deren Fähigkeiten der Begriff von einer allgemeinen Harmonie des Weltgebäudes noch viel zu hoch ist, als daß sie daraus den Schöpfer gehörig erkennen sollten. Übrigens ist wohl nichts augenscheinlicher und gewisser, als daß die Zusätze, die auf dieser Reise zum Ganzen der menschlichen Kenntnisse gemacht worden, obschon nicht ganz unbeträchtlich, dennoch von geringem Werth sind, sobald wir sie mit dem, was uns noch verborgen bleibt, in Vergleichung stellen. Unzählig sind die unbekannten Gegenstände, welche wir, aller unsrer Einschränkung ohngeachtet, noch immer erreichen können. Jahrhunderte hindurch werden sich noch neue, unbeschränkte Aussichten eröfnen, wobey wir unsere Geisteskräfte in ihrer eigenthümlichen Größe anzuwenden, und in dem herrlichsten Glanze zu offenbaren Gelegenheit finden werden. ${ }^{9}$

So endet also Georg Forsters erfolgreiche und überaus populäre, eine wahre Südseetrunkenheit beim deutschsprachigen Publikum auslösende Darstellung

9 Forster, Georg: Reise um die Welt, S. $997 \mathrm{f}$. 
der zweiten Reise von James Cook in einem panoramatischen Kurzüberblick über die Verschiedenheit des Menschengeschlechts auf unserem Planeten. Es impliziert ohne jeden Zweifel die herausragende Rolle des europäischen Teiles der Menschheit und definiert alle Völkerschaften mit Blick auf diesen 'natürlichen' Bezugspunkt. Hier von Eurozentrismus zu sprechen, verbietet sich fast, erscheint er doch als evident.

Aber hier liegt nicht der Kern des Denkens und der weitgespannten Überlegungen Georg Forsters. Wir finden in diesen Zeilen vielmehr das Element einer abschließenden philosophischen Rahmung, wie wir es auch später bei Georg Forsters 'Schüler' Alexander von Humboldt in Vollendung sehen werden. Schon Georg Forster ging es vor allem darum, das Ganze im Auge zu behalten, sich nicht an einzelnen Elementen und Details festzuklammern, sondern ein Gesamtbild der Menschheit wie auch des ihr anvertrauten Planeten zu entwerfen, das - durch viele einzelne Details und Feldforschungen untermauert stets eine panoramatische und historische Perspektive zugleich in Raum und Zeit entfalten sollte. Dabei wird deutlich, wie sehr der Weltreisende die Vorläufigkeit seiner Erkenntnisse betont und deutlich macht, wieviel es noch jahrhundertelang auf den unterschiedlichsten Gebieten zu erforschen gebe. Der reiseliterarische Ort der Rückkunft wird zum Ort der Verständigung über das wissenschaftlich Geleistete und das noch wissenschaftlich zu Leistende. Erkenntnis - und zumal philosophische Erkenntnis - ist ein unabschließbarer, nie zu Ende zu bringender Prozess.

Unschwer erkennen wir in dieser Passage bei allem zeithistorischen Verständnis aber auch, dass wir es hier mit einer Weltsicht und einer Weltanschauung des 18. Jahrhunderts zu tun haben, die im Grunde ganz 'natürlich' und selbstverständlich von Europa aus und für Europa denkt, das Menschengeschlecht andernorts also in einer hierarchischen Beziehung gegenüber den europäischen Kulturvölkern versteht und dabei vor allem das Abweichen, die Devianz der Anderen beziehungsweise des Anderen betont. In einem Atemzug werden zum einen die Einheit des Menschengeschlechts und dessen Variantenreichtum betont, wobei letzterer in ein vorzeitliches Verhältnis zu den Völkern Europas gesetzt wird. Historischer Fluchtpunkt aber ist und bleibt Europa.

Eben dies ist für Forsters Bericht von seiner Reise um die Welt entscheidend. Dabei ist aufschlussreich, dass der Mensch an anderen Orten unserer Erde - etwa in Feuerland - gleichsam auf der Stufe des Tieres zu vegetieren scheint, also bestimmter Formen des Menschentums an sich - das universal gültig gesetzt wird - nicht teilhaftig ist. Hierzu zählt Forster vor allem das gesellige (und damit gesellschaftliche, arbeitsteilige) Leben sowie bestimmte Werte, welcher die 'Anderen' - wie schon bei La Condamine - nicht teilhaftig sind, welche diese Völker nicht besitzen und sie nicht zu kümmern scheinen, ja 
mehr noch: Diese Völkerschaften haben nicht einmal Wörter dafür. Somit ist auch noch bei Georg Forster, aller Offenheit zum Trotz, alles an einem Bild des Menschen und der Menschheit ausgerichtet, das unverkennbar europäisch und abendländisch geprägt ist. Es ist die Ausdifferenzierung gesellschaftlicher Zustände, die Komplexität im Sinne einer arbeitsteiligen abendländischen Gesellschaft, welche im Zentrum der Beurteilungen Georg Forsters steht, und nicht etwa die Frage nach dem Glück des Menschen, die einen universal gültigen Wert für alle Varietäten, für alle Kulturen des Menschen darstellen würde.

Zugleich werden alle Entwicklungsstufen in ein Noch-Nicht überführt, so dass - wie in Friedrich Schillers Jenaer Antrittsvorlesung 'Was ist und zu welchem Ende studiert man Universalgeschichte?' - letztlich alle anderen Völker um uns her gelagert sind und ihre Berechtigung eigentlich erst als Verkörperungen von Entwicklungsständen gewinnen, die sie auf dem Weg zum abendländischen Menschen, dem Menschen par excellence, repräsentieren. Die Entwicklung des abendländischen Wissens, aber auch des abendländischen Menschen wird zugleich als prinzipiell offen, als eine sich ständig neu vollziehende Progression gesehen, an deren Anfang wir heute erst stünden. Damit wird auch die Reise von Forster selbst in ein umfassendes Bild integriert und zu einem Teil der Entwicklungsgeschichte der Menschheit stilisiert. Jeder einzelne Aspekt, jedes einzelne Detail und damit letztlich auch das gesamte menschheitsgeschichtliche Portrait selbst werden in eine historische Zeitlichkeit versetzt, in eine Geschichte, deren Zeitpfeil unumkehrbar ist. Die Einführung der Zeit - einer unumkehrbaren, nicht länger zyklisch, sondern linear verlaufenden Zeit - ist in Georg Forsters Reise um die Welt integriert und eröffnet damit ein Entwicklungsgemälde, das kein Tableau mehr im klassischen Sinne darstellt, sondern im Sinne der Moderne von der Zeit untersetzt ist. Im letzten Viertel des 18. Jahrhunderts können wir anhand dieses Reiseberichts unverkennbar den Übergang der Reiseliteratur in die Moderne konstatieren. Es ist eine europäische Moderne.

In der aufklärerischen, noch ganz dem 18. Jahrhundert verpflichteten Einfärbung dieser Moderne deutet sich ein Fortschrittsoptimismus an, der letztlich auch die Wahrnehmung und Darstellung der Reise selbst betrifft. Denn es ist die Entdeckungs- und Forschungsreise wie jene, an welcher Forster selbst teilnehmen durfte, die eine wichtige Funktion innerhalb der skizzierten menschheitsgeschichtlichen Entwicklung übernimmt, indem sie ein weiteres Fortschreiten des Menschen im Wissen über sich selbst gewährleisten soll. So ist der Reisebericht Instrument der Reflexion und der Erkenntnis, der Sammlung und Entwicklung von Wissen, die Repräsentationsform einer Bewegung also, die der Reisebericht zugleich verkörpert und demonstriert, präsentiert und repräsentiert. 
Innerhalb einer derartigen Entwicklung kommt dem (literarischen) Reisebericht eine ungeheure Wichtigkeit und Bedeutung zu. An einer solchen Auffassung aber partizipierte auch noch jener wohl bekannteste deutschsprachige Forschungsreisende, der in seiner Jugend dank einer gemeinsamen Reise mit Georg Forster das wohl entscheidende reiseliterarische Bildungserlebnis erfuhr und fortan nicht mehr zu Hause zu halten war. Wenden wir uns also am Übergang des 18. zum 19. Jahrhundert Alexander von Humboldt und seinem Verständnis von Reiseliteratur zu. 